\title{
Factores de crecimiento relacionados con la activación de folículos primordiales en bovinos
}

\section{Growth factors related to the activation of primordial follicles in cattle}

\author{
Lidy Viviana Castillo Barón ${ }^{1}$ \\ E-mail: vivicastillo5@hotmail.com \\ Universidad Nacional Abierta y a Distancia (UNAD) \\ ${ }^{1}$ Escuela de Ciencia Agrarias y del Medio Ambiente (ECAPMA)
}

Resumen.- Se analizó la presencia y expresión de moléculas denominadas factores de crecimiento, que actúan controlando la dinámica folicular en etapas tempranas; además se describió la naturaleza y mecanismo de acción de los principales factores de crecimiento que intervienen en la activación de los folículos primordiales en bovinos, para mostrar la importancia en el desarrollo de folículos y oocitos de calidad que puedan ser fecundados, aumentando el potencial reproductivo de los animales domésticos.

Palabras clave: Factores de crecimiento, folículo, oocito, bovino.

Abstract.- This article analyzes the presence and expression of molecules, called growth factors that act to control follicular dynamics in early stages; It also describes the nature and mechanism of action of the major growth factors involved in the activation of the paramount follicles in cattle. This is to show the importance of these molecules in developing follicle and oocyte quality, which can become fertilized on the way to increase the reproductive potential of domestic animals.

Key words: Growth factors, follicle, oocyte, bovine.

\section{Introducción}

En bovinos, la unidad funcional del ovario es el folículo y se compone de un oocito en desarrollo rodeado por una o varias capas de células somáticas (Fair et al., 1997) que son el sitio de acción y síntesis de gran número de hormonas y factores de crecimiento que promueven y regulan el desarrollo folicular, gracias a un sistema de comunicación bidireccional mediante la transferencia de señales locales y endocrinas que se establece con las células somáticas y el oocito (Eppig, 2001; Gilchrist et al., 2004; Skinner, 2005).

La función de las moléculas asociadas con la activación de los folículos primordiales en bovinos, explica la importancia de iniciar el crecimiento de este grupo de folículos en reposo y 
mantenerlo hasta una etapa en la que el oocito madure y sea fecundado. De esta manera se evalúa la calidad de los folículos y sus oocitos, los cuales son parte importante en la aplicación de las biotecnologías de la reproducción y de la conservación en especies en peligro de extinción y en mujeres infértiles (Fortune et al., 1998; Fortune, 2003).

\section{Folículos primordiales}

En los últimos años la investigación sobre la dinámica folicular se ha centrado cada vez más en el desarrollo folicular temprano, concretamente, en la etapa preantral - primordial (Aerts \& Bols, 2008). Un folículo primordial consta de un oocito cuyo crecimiento se ha detenido en el estado de diploteno de la profase I de la meiosis y está rodeado por una capa simple de células planas (pregranulosas) constituyendo la reserva de folículos en reposo (Nilsson et al., 2001).

En algunas especies como los roedores, la reserva folicular se establece en el momento del nacimiento, mientras que en los primates y especies domésticas se desarrolla durante la vida fetal, y la transición a folículos en crecimiento es un proceso gradual que comienza poco después de la formación de los folículos primordiales y continúa durante toda la vida reproductiva (Aerts \& Bols, 2008; Fortune et al.,1998).

\section{Característicasy MecanismodeAcción de los Factores de Crecimiento}

La activación, crecimiento inicial de los folículos primordiales y posterior desarrollo folicular está comandada por factores de crecimiento intraováricos de acción local no dependientes de gonadotropinas (Wezel \& Rodgers, 1996; Fortune, 2002; Palma, 2008; Aerts \& Bols, 2008).

Los factores de crecimiento son polipéptidos semejantes a las hormonas, de peso molecular $<30.000 \mathrm{Kd}$. Son producidos a partir de diversos tipos celulares y no se almacenan intracelularmente (Hill, 1989). Estos factores ejercen su acción a nivel local de forma autocrina y paracrina, para lo cual requieren la comunicación bidireccional entre el oocito y las células granulosas y simultáneamente entre las células granulosas y el oocito (Eppig, 2001). La unión celular que permite la comunicación bidireccional se conoce como gap junction, constituida por proteínas conocidas como conexinas (Fair, 2003) (Figura 1).

Estas proteínas forman un poro que permite el paso de moléculas en doble dirección del citoplasma de una célula al citoplasma de otra sin pasar por el espacio extracelular. Este modo de comunicación es esencial para el desarrollo y la fertilidad ya que el oocito dirige la proliferación, diferenciación y función de células de la granulosa, mientras que las células de la granulosa realizan una tarea de nutrición y mantenimiento del oocito permitiendo el paso de iones, metabolitos, aminoácidos y pequeñas moléculas necesarias para su crecimiento (Aerts \& Bols, 2008). 


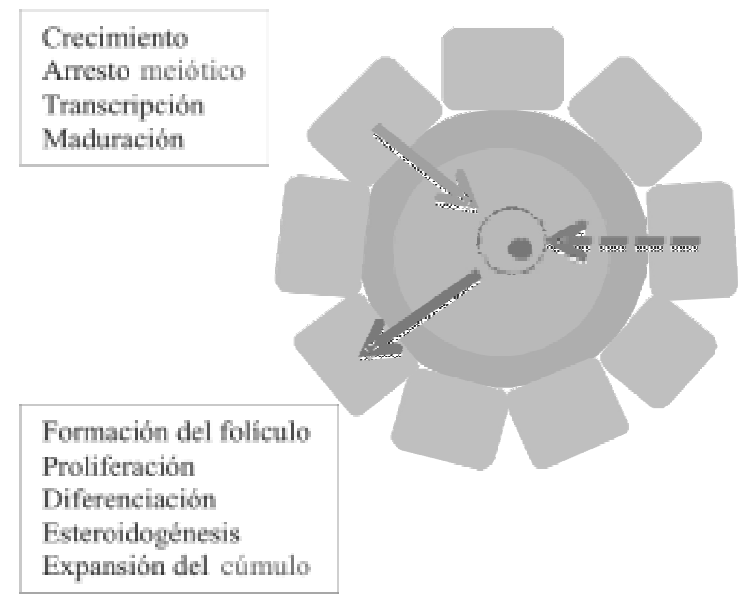

Figura 1. Comunicación bidireccional entre el oocito y el conjunto de células somáticas e influencia mutua entre células granulosas y desarrollo del oocito.

\section{Activación de Folículos Primordiales}

La activación de los folículos primordiales comprende el paso de un estado de reposo a una fase de crecimiento caracterizada por dos fases consecutivas: (I) la proliferación y cambio de forma de las células granulosas (de planas a cuboides) y (II) El incremento en el diámetro del oocito (Wezel \& Rodgers, 1996; Braw-Tal, 2002).

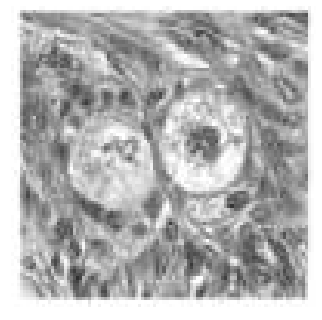

$2 \mathrm{a}$

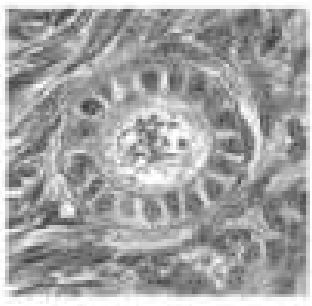

$2 b$

Figura 2. Etapas del crecimiento folicular ovárico en bovinos: (2a) Folículo primordial; (2b) Folículo primario. (Tomado de Biotecnología de la Reproducción. Palma, 2008).

Los factores que estimulan la proliferación y transformación (de planas a cuboidales) de las células pregranulosas son considerados probables promotores de la activación de folículos primordiales, ya que parece que un requisito para el 
inicio del crecimiento de los oocitos es la presencia de células de la granulosa cúbica (Braw-Tal, 2002). El papel del oocito en la activación de los folículos primordiales está determinado por la acción del figla, también llamado Fig $\alpha$. Se trata de un gen que coordina por lo menos dos acciones en el oocito: (I) la expresión de genes estructurales de proteínas que componen la zona pelúcida y (II) la producción de uno o más factores esenciales para la organización de folículos primordiales (Eppig, 2001).

Adicionalmente, dos miembros de la superfamilia del factor de crecimiento transformante $\beta$ (TGF- $\beta$ ), el factor de crecimiento y diferenciación 9 (GDF-9) y la proteína morfogénica de hueso 15 (BMP-15) son expresadas de manera específica en el oocito en estadios tempranos y juegan un papel importante promoviendo el crecimiento folicular hacia el estadio primario (Knight \& Glister, 2003).

Nilsson et al. (2001) demostraron por medio de cultivos de ovario neonatal de ratas, la participación del factor de crecimiento fibroblástico (FGF, FGF-2 también llamado FGF básico) en el desarrollo folicular temprano. Se encontró que el FGFb expresado por el oocito de los folículos primordiales y primarios, regula la expresión del ligando Kit (KL) y promueve el crecimiento de las células de la granulosas, las células tecales y el estroma durante el desarrollo de folículos (Nilsson et al., 2001).

Por otro lado, se ha encontrado que las células pregranulosas que rodean el oocito de los folículos primordiales expresan factores peptídicos como el ligando Kit (KL) y el factor inhibidor de la leucemia (LIF) que actúan de forma paracrina/autocrina promoviendo la transición del folículo primordial a primario in vitro (Figura 2). Estos factores estimulan el crecimiento del oocito y la proliferación de las células del estroma que rodea el folículo (Nilsson et al., 2002). Los receptores para KL (c-kit) son expresados por el oocito y por las células mesenquimales intersticiales permitiendo la respuesta para estos factores de crecimiento.

Algunas de las células mesenquimales que rodean los folículos primordiales (precursoras de células tecales) han sido programadas para producir otro péptido llamado factor de crecimiento de los keratinocitos -KGF (o factor de crecimiento fibroblástico-7, FGF-7). Es probable que este actúe sobre las células pregranulosas regulando su proliferación y amplifique el efecto positivo sobre las células pretecales y sobre el crecimiento del oocito. Lo anterior fue demostrado en cultivos de ovarios de ratas sometidos a distintos tratamientos en los que se incluyó KGF (100 ng/ml) que fue localizado inmunohistoquímicamente en las células del estroma adyacentes a la capa de células granulosas del folículo primordial en crecimiento (Kezele et al., 2005).

En cultivos de ovario de rata se encontró que la presencia de BMP-7 promovía la división celular y la proliferación de células granulosas, evento fundamental para la activación de folículos primordiales. Por tanto, el desarrollo folicular temprano depende de la interacción de factores como LK, 
GDF-9, BMP-15, FGFb (Figura 3), desencadenando una serie de eventos que resultan en el inicio del proceso de foliculogénesis.

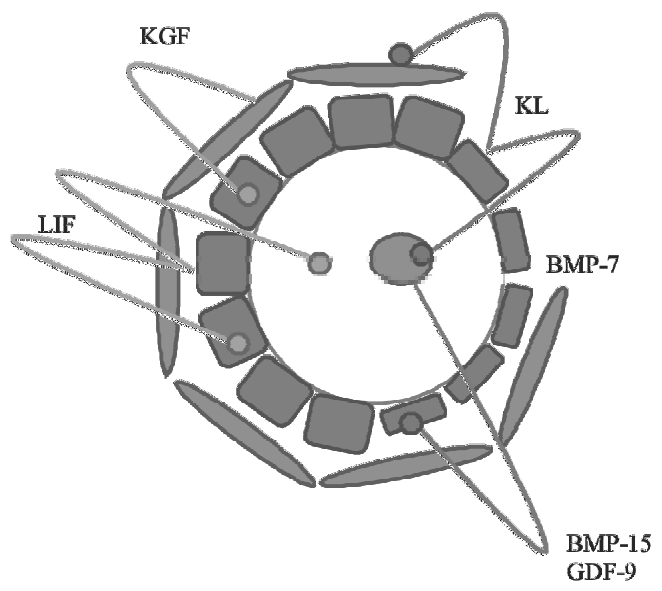

Figura 3. Esquema de interacción celular que coordina la transición de folículo primordial a primario.

\section{Conclusiones}

Durante el desarrollo folicular temprano intervienen diversas proteínas y polipéptidos que conforman el sistema de factores de crecimiento y aunque no se conoce con exactitud el mecanismo de acción de estas moléculas, numerosos estudios han identificado su presencia en las células de la granulosa, las células tecales y el oocito, ejerciendo acción estimulatoria en los diferentes estadios de desarrollo.

La acción de los factores de crecimiento es ejercida a nivel local por medio de un sistema de comunicación entre el oocito y las células granulosas y a su vez entre las células granulosas y las células tecales. Los principales factores de crecimiento que intervienen en la activación de los folículos primordiales son: LK, GDF-9, BMP-15 y FGFb. Estas moléculas actúan desencadenando una serie de eventos que desembocan en el inicio del proceso de foliculogénesis.

\section{Recomendaciones}

Esta revisión evidencia la necesidad de mejorar la comprensión de las etapas tempranas del desarrollo folicular en los rumiantes domésticos. Se recomienda continuar investigando sobre los mecanismos que regulan la activación y el crecimiento de folículos primordiales hasta una etapa en la que el oocito madure y sea fecundado, aumentando el potencial reproductivo de valiosos animales domésticos.

\section{Referencias}

1) Aerts, J. \& Bols, P. (2008). Ovarian Follicular Dynamics: A Review with Emphasis on the Bovine Species. Part I: Folliculogenesis and Pre-antral Follicle Development. Reproduction 
in Domestic Animals, (45), 171-179. Recuperado de http://onlinelibrary. wiley.com/doi/101111/j.1439. 0531.2008.01302.x/abstract .

2) Braw-Tal, R. (2002). The initiation of follicle growth: The oocyte or the somatic cells? Molecular and Cellular Endocrinoly, (187), 1118. Recuperado de http://www. sciencedirect.com/science/article/pii/ S0303720701006992

3) Eppig, J. (2001). Oocyte control of ovarian follicular development and function in mammals. Reproduction, (122), 829-838. Recuperado de http://www.reproduction-online.org/ content/122/6/829.short

4) Fair, T. (2003). Follicular oocyte growth and acquisition of developmental competence. Animal Reproduction Science, (78), 203-216. Recuperado de http://mvz.unipaz. edu.co/textos/revistas/literatura-1/ foliculos-competencia.pdf

5) Fair, T., Hulshof, C. J. Hyttel, P., Greve, T. \& Boland, M. (1997). Oocyte ultrastructure in bovine primordial to early tertiary follicles. Anatomy and Embryology, (195), 327-336. Recuperado de http://http:// mendeley.com/research/oocyteultrastructure-bovine-primordialearly-tertiary-follicles/

6) Fortune, J. (2002). Activation of primordial follicles. Recuperado de http://mendeley.com/research/ activation-bovine-baboonprimordial-follicles-vitro/
7) Fortune, J. (2003). The early stages of follicular development: Activation of primordial follicles and growth of preantral follicles. Animal Reproduction Science, (78), 135-163 Recuperado de http://mvz.unipaz. edu.co/textos/revistas/literatura-1/ desarrollo-folicular.pdf.

8) Fortune, J., Kito, S., Wandji, S. A. \& Srsen, V. (1998). Activation of bovine and baboon primordial follicles in vitro. Theriogenology, (49), Suppl 2, 441-449.

9) Gilchrist, R., Ritter, L. \& Armstrong, D. (2004). Oocytesomatic cell interactions during follicle development in mammals. Animal Reproduction Science. Recuperado de http://www. sciencedirect.com/science/article/ pii/S0378432004001083.

10)Hill, D. J. (1989). Growth factors and their cellular actions. Journal of Reproduction \& Fertility, (85), 723734. Doi: 10.1530/jrf.0.0850723.

11)Kezele, P., Nilsson, E. \& Skinner, M. (2005). Keratinocyte growth factor acts as a mesenchymal factor that promotes ovarian primordial to primary follicle transition. Biology of Reproduction, (73), 967-973. Recuperadodehttp://www.biolreprod. org/content/73/5/967.ful.pdf.

11)Knight, P. \& Glister, C. (2003). Local roles of TGF- $\beta$ superfamily members in the control of ovarian follicle development. Animal Reproduction Science, (78), 165-183. Recuperado de http://mvz. unipaz.edu.co/textos/revistas/literatura-1/ ovarios-foliculos.pdf. 
12)McGee, E. \& Hsueh A. J. (2000). Initial and cyclic recruitment of ovarian follicles. Endocrine Reviews, (21), Suppl 2, 200-214. Recuperado de http://edrv.endojournals.org/ content $/ 21 / 2 / 200$.abstract.

13)Nilsson, E., Kezele, P. \& Skinner, M. (2002). Leukemia inhibitory factor (LIF) promotes the primordial to primary follicle transition in rat ovaries. Molecular and Cellular Endocrinology, (188), 65-73. Recuperado de http://ncb.nlm.nib. gov/pubmed/11911947.

14)Nilsson, E., Parrott, J. \& Skinner, M. (2001). Basic fibroblast growth factor induces primordial follicle development and initiates folliculogenesis. Molecular and Cellular Endocrinology, (175), 123130. Recuperado de http://skinner. wsu.edu/members/nilsson.html.

15)Palma, G. \& Brem, G. (2001). Biotecnología de la Reproducción.
En G. Palma, Biotecnología de la reproducción, cap. 1, 1-19. Buenos Aires, Argentina: Ediciones Instituto Nacional de Tecnología Agropecuaria.

16)Skinner, M. (2005). Regulation of primordial follicle assembly and development. Human Reproduction, 11 (5), 461-471. Recuperado de http://humupd.oxfordjournals.org/ content/11/5/461.abstract.

17)Wezel, I. L. \& Rodgers, R. J. (1996). Morphological characterization of bovine primordial follicles and their environment in vivo. Biology of Reproduction, (55), 10031011. Recuperado de http://www. biolreprod.org/content/55/5/1003. full.pdf.

Recibido: 23 de septiembre de 2011 Aceptado: 30 de noviembre de 2011 